\title{
Reviewer Acknowledgements for Global Journal of Health Science, Vol. 10, No. 7
}

Global Journal of Health Science wishes to acknowledge the following individuals for their assistance with peer review of manuscripts for this issue. Their help and contributions in maintaining the quality of the journal are greatly appreciated.

Global Journal of Health Science is recruiting reviewers for the journal. If you are interested in becoming a reviewer, we welcome you to join us. Please find the application form and details at http://recruitment.ccsenet.org and e-mail the completed application form to gjhs@ccsenet.org.

\section{Reviewers for Volume 10, Number 7}

Abdulbari Bener, Istaanbul University, Turkey

David Otieno Odongo, Masinde Muliro University of Science and Technology, Kenya

Darampal Dambhare, Mahatma Gandhi Institute of Medical Sciences, India

Dilek Pirim, Department of Molecular Biology and Genetics, Uludag University, Turkey

Domitila Augusta Huber, Federal University of Santa Catarina, Brazil

Emad Adel Shdaifat, Imam Abdulrahman Bin Faisal University, Saudi Arabia

Farahnaz Amini, UCSI University, Malaysia

Hadii M Mamudu, East Tennessee State University, United States

Jaime Hinzpeter, Clinical Hospital University of Chile, Chile

Jason Tsai, Lincoln College, United Kingdom

Jingxian Cai, ICSA, ASA, ENAR, United States

José Joaquín Mira, Universidad Miguel Hernández, Spain

Jose R Cordon, Universidad de Cadiz (Spain);SASEMAR(M. Fomento-Spain)-Spanish Goverment, Spain

Kartheek R Balapala, University Tunku Abdul Rahman, Malaysia

Dr Kim Solez, University of Alberta, Canada

Krzysztof Goniewicz, Medical University of Warsaw; Polish Air Force Academy, Poland

Marcel Wullschleger, Department of Physiology, University of Bern, Switzerland

Marcelle Bottecchia, Instituto Oswaldo Cruz - FIOCRUZ, Brazil

Mini Sood, Monash University Malaysia, Malaysia

Meng Zhao, Texas A\&M University at Corpus Christi, United States

Montarat Thavorncharoensap, Mahidol University; Health Intervention and Technology Assessment Program (HITAP), Ministry of Public Health, Thailand

Myo Nyein Aung, Juntendo University, Japan; Boromrajonani College of Nursing, Thailand

Natasha AZZOPARDI MUSCAT, University of Malta, Maastricht University, Malta

Soon Soo Hoo, Royal North Shore Hospital, Sydney, Australia

Srikrishna Sulgodu Ramachandra, Public Health Foundation of India, India

Thanusin Saleeon, Nan Provincial Public Health Office, Ministry of Public Health, Thailand

Thammanard Charernboon, Thammasat University, Thailand

Trisha Dunning, Deakin University and Barwon Health, Australia

Tsan Yang, Meiho University, Taiwan, Province of China 